\title{
ARTICLE \\ A homolog of cyclophilin D is expressed in Trypanosoma cruzi and is involved in the oxidative stress-damage response
}

\author{
Patricia L Bustos ${ }^{1,2}$, Bibiana J Volta ${ }^{1,4}$, Alina E Perrone ${ }^{1}$, Natalia Milduberger ${ }^{1,3}$ and Jacqueline Bua ${ }^{1,2,3}$
}

\begin{abstract}
Mitochondria have an important role in energy production, homeostasis and cell death. The opening of the mitochondrial permeability transition pore (mPTP) is considered one of the key events in apoptosis and necrosis, modulated by cyclophilin D (CyPD), a crucial component of this protein complex. In Trypanosoma cruzi, the protozoan parasite that causes Chagas disease, we have previously described that mitochondrial permeability transition occurs after oxidative stress induction in a cyclosporin A-dependent manner, a well-known cyclophilin inhibitor. In the present work, a mitochondrial parasite cyclophilin, named TcCyP22, which is homolog to the mammalian CyPD was identified. TcCyP22-overexpressing parasites showed an enhanced loss of mitochondrial membrane potential and loss of cell viability when exposed to a hydrogen peroxide stimulus compared with control parasites. Our results describe for the first time in a protozoan parasite that a mitochondrial cyclophilin is a component of the permeability transition pore and is involved in regulated cell death induced by oxidative stress.
\end{abstract}

Cell Death Discovery (2017) 3, 16092; doi:10.1038/cddiscovery.2016.92; published online 6 February 2017

\section{INTRODUCTION}

Mitochondria serve as the 'powerhouse' that provides near $90 \%$ of ATP necessary for cell life. However, studies over the past 30 years have provided strong evidence that mitochondria also have a central role in cell death in response to oxidative stress. ${ }^{1}$ Accumulation of reactive oxygen species (ROS) together with $\mathrm{Ca}^{2+}$ overload induces the mitochondrial permeability transition (mPT), which indicates an abrupt increase in the permeability of the inner mitochondrial membrane to small solutes. ${ }^{2,3}$ In this setting, osmotic forces drive a massive entry of water into the mitochondrial matrix, leading to an immediate cessation of the bioenergetics and biosynthetic functions of mitochondria that depend on the transmembrane potential $\left(\Delta \Psi_{\mathrm{m}}\right)$ and the release of multiple mitochondrial proteins, including various activators of the intrinsic pathway of apoptosis into the cytoplasm. ${ }^{4}$

The occurrence of $\mathrm{mPT}$ is the key event of the regulated cell death process. The term 'regulated' refers to cases that can be inhibited by specific pharmacological or genetic interventions, implying that they rely on defined molecular machinery, though sometimes known to partial extents. Until recently, apoptosis was considered as the only form of regulated cell death but since late 1990 s it has become clear that necrosis can occur in a regulated fashion, known as regulated necrosis (RN). For example, the mPT represents one of the major gateways to mitochondrial apoptosis, a process that is mediated and regulated by proteins of the $\mathrm{Bcl}-2$ family, namely, mitochondrial outer membrane permeabilization (MOMP). ${ }^{5,6}$ However, the mPT is also capable of triggering a peculiar process of $\mathrm{RN}$ that critically relies on peptidyl prolyl isomerase F (PPIF, best known as cyclophilin D, CyPD), which results in the opening of a non-selective pore, namely the mitochondrial permeability transition pore (mPTP). The importance of CyPD for MPT has been recognized for a long time mostly due to the consistent cytoprotective effects mediated in vitro and in vivo by the pharmacological CyPD inhibitor cyclosporin $\mathrm{A}$ $(\mathrm{Cs} A)^{2,3}$ Nowadays, CyPD remains the only confirmed functional constituent of the mPTP.

Moreover, both the administration of CsA and the Ppif ${ }^{-1}$ genotype have been shown to limit necrotic cell death, in vitro as well as in vivo, in a variety of pathophysiological settings including ischemia-reperfusion injuries of the heart, ${ }^{7-9}$ brain $^{10,11}$ and kidneys. ${ }^{12,13}$ Interestingly, the pro-necrotic activity of CyPD appears to be regulated by a mitochondrial pool of the oncosupressor protein p53. ${ }^{11}$ However, the precise molecular mechanisms that execute mPT-dependent RN is still obscure. It remains to be formally demonstrated whether CyPD mediates RN as part of the MPTP or independently from the assembly of such a protein complex. As mentioned above, so far no MPTP components other than CyPD have been shown to be indispensable for $\mathrm{mPT}$ in vivo. This may indicate that CyPD can assemble the mPTP in a relatively unspecific manner or that CyPD exerts MPTPindependent lethal functions.

Trypanosoma cruzi is a unicellular protozoan parasite which infects 7-8 million people in South America as well as in other parts of the world through migrations from endemic areas. ${ }^{14}$ The $T$. cruzi infection can evolve into Chagas disease, a potential lifethreatening illness. ${ }^{15}$

We have previously described the $T$. cruzi CyP gene family, which consists of 15 paralogs. ${ }^{16,17}$ Analyses of clusters formed by the $T$. cruzi CyPs with others encoded in various genomes revealed that eight parasite cyclophilins (TcCyP19, TcCyP21, TcCyP22, TcCyP24, TcCyP35, TcCyP40, TcCyP42 and TcCyP110) have orthologs in many different genomes (reviewed in Galat and Bua $\left.^{18}\right)$. Moreover, 4 T. cruzi CyPs $(19,22,28$ and $40 \mathrm{kDa}$ proteins) were isolated by $\mathrm{CsA}$ affinity from parasite lysates that proved to have PPlase activity, inhibited by CsA. ${ }^{16,17}$

We have also described a regulated cell death process occurring under oxidative stress conditions in $T$. cruzi, including features as

\footnotetext{
${ }^{1}$ Instituto Nacional de Parasitología ‘Dr. Mario Fatala Chabén'- A.N.L.I.S. Malbrán, Av. Paseo Colón 568, C1063AC S, Buenos Aires, Argentina; ${ }^{2}$ Consejo Nacional de Investigaciones Científicas y Técnicas, Argentina and ${ }^{3} \mathrm{CAECIHS}$, Universidad Abierta Interamericana, Av. Montes de Oca 745, $2^{\circ}$ piso, C1270AAH, Buenos Aires, Argentina.

Correspondence: Dr PL Bustos (pato54mar@yahoo.com.ar)

${ }^{4}$ Present address: Laboratorio de Nutrición Animal, INTA Ruta Nac N 9 Km 1108, Santiago del Estero, Argentina.

Received 19 August 2016; revised 11 October 2016; accepted 24 October 2016; Edited by N Berlev
} 
phosphatidyl serine exposure, DNA degradation and cytochrome $c$ translocation into the cytosol, among others. All these events were sensitive to CSA inhibition, suggesting that a T. cruzi mitochondrial cyclophilin could be present in a mPTP-like in this protozoan. ${ }^{19}$

In the present work, we demonstrate the existence of mitochondrial cyclophilin, TCCyP22, homolog to the mammalian CyPD, in T. cruzi and described its specific role in parasite cell death in response to oxidative stress.

\section{RESULTS}

A mitochondrial cyclophilin is expressed in T. cruzi

We have previously described that some cell death features induced by oxidative stress could be inhibited by cyclosporin A in Trypanosoma cruzi, thus indicating that a parasite mitochondrial CyP may be involved in these events. ${ }^{19}$

To predict the CyPs with putative mitochondrial localization within the parasite cyclophilin family, we performed a bioinformatic analysis, combining the information obtained from analyzing the amino acid sequences of the CyP family with two different servers, iPSORT and MitoProtll v1.101 (see Material and Methods section). The parameters obtained for each CyP are listed in Supplementary Table S1. We observed that TcCyP21, TcCyP22, TCCyP24 and TcCyP25 presented high probability of being localized to $T$. cruzi mitochondrion.

When we aligned these protein sequences with human CyPD CyPD (GenBankAcc No. P30405), TcCyP22 (GenBankAcc No. AY349021.1/TriTryP DB Acc No. TcCLB.510259.50) presented the highest percentage of amino acid identity (65\%) and similarity (77\%) (Supplementary Figure S1).

Since some cross-reactivity might appear with antibodies against conserved regions of cyclophilins in indirect immunofluorescence techniques, we developed a molecular biology strategy to confirm the TcCyP22 localization. The complete DNA sequence was amplified by PCR and cloned with a C-terminal enhanced version of the green fluorescent protein (eGFP) in the pTREX vector. A transgenic parasite line carrying this construction was generated, hereinafter referred to as TCCyP22-OE. Immunofluorescence was performed with monoclonal antibodies against GFP in TCCyP22-OE in the epimastigote stage. TcCyP22 localized to the mitochondrion, as demonstrated by co-localization with MitoTracker staining (Figure 1a). To investigate if TcCyP22 was expressed in the other stages of the parasite lifecycle, we infected VERO cells. We obtained transgenic parasites in the amastigote and tripomastigote stages. We observed that TcCyP22 was expressed and localized to the mitochondrion of both stages as well (data not shown).

Protein size was confirmed by Western blot analysis of total parasite lysates, where a unique band was detected in each lane, using antibodies against GFP ( 40 kDa for TcCyP22-GFP and $\sim 20 \mathrm{kDa}$ for GFP; Figure 1b).

TcCyP22-overexpressing parasites are more susceptible to oxidative stress damage

As we intended to use the transgenic parasites in the epimastigote stage for further studies, we first attempted to evaluate if the overexpression of TcCyP22 altered parasite viability. We confirmed that there were no significant differences in the number, motility and morphology between TcCyP22-OE and wild type parasites (data not shown).

Since the specific mitochondrial cyclophilin modulating these events remained unknown, we challenged TCCyP22-OE parasites in oxidative stress conditions to analyze if TCCyP22 is involved in cell death.

TcCyP22-OE shows enhanced loss of mitochondrial membrane potential $\left(\Delta \Psi_{\mathrm{m}}\right)$

The loss of mitochondrial membrane potential $\left(\Delta \Psi_{\mathrm{m}}\right)$ occurs at the onset of cell death process. To study the $\Delta \Psi_{\mathrm{m}}$ of TcCyP22-OE in oxidative stress conditions, parasites were loaded with the mitochondrial fluorescent $\Delta \Psi \mathrm{m}$ indicator MitoTracker. Parasites carrying the empty pTREX-GFP vector were used as control. After oxidative stress induction, transgenic epimastigotes were analyzed by flow cytometry to evaluate the fluorescence intensity, which was considered proportional to the $\Delta \Psi_{\mathrm{m}}$ of the parasite population at different time points.

The fluorescence measured at the beginning of the induction with $\mathrm{H}_{2} \mathrm{O}_{2}$ (time $0 \mathrm{~min}$ ) was considered as $100 \% \Delta \Psi_{\mathrm{m}}$ (in arbitrary units). After $15 \mathrm{~min}$, a substantial decrease to $43.26 \%$ was observed in TcCyP22-OE parasites. In contrast, $\Delta \Psi_{\mathrm{m}}$ in control parasites (TREX), at $15 \mathrm{~min}$ lowered only to $86.9 \%$. When measured at $60 \mathrm{~min}$, loss of $\Delta \Psi_{\mathrm{m}}$ was enhanced in TcCyP22-OE compared with TREX parasites, decreasing to 21.0 and $43.26 \%$, respectively (Figure 2). TCCyP22-OE parasites evidenced an enhanced loss of $\Delta \Psi \mathrm{m}$ compared with control parasites in oxidative stress conditions.

The genomic DNA of TCCyP22-OE parasites results highly damaged after oxidative stress induction

To further characterize the damage caused by oxidative stress in TcCyP22-OE parasites compared with control parasites, we evaluated the integrity of genomic DNA after the oxidative stress with hydrogen peroxide by terminal deoxynucleotidyltransferase dUTP nick end labeling (TUNEL).

We evaluated the percentage of positive TUNEL-stained parasites at 60 and $180 \mathrm{~min}$ after oxidative stress. At $60 \mathrm{~min}$, we observed that TcCyP22-OE presented a significantly higher a

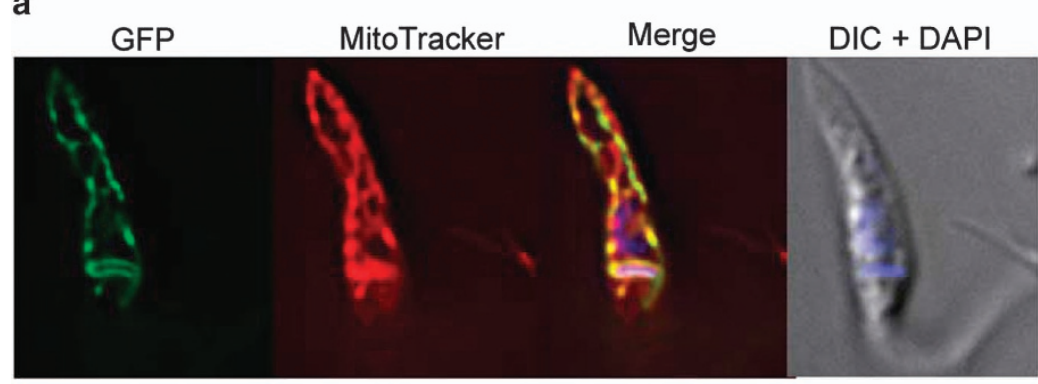

b

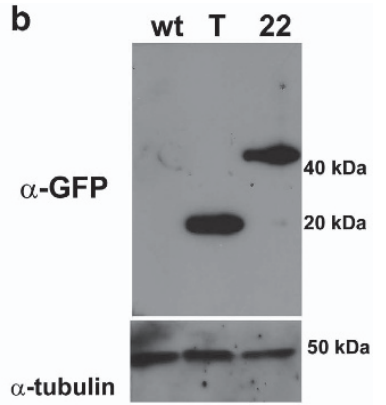

Figure 1. Expression and subcellular localization of TcCyP22 in transgenic epimastigotes. (a) Monoclonal antibodies against GFP were used to detect TcCyP22 (green). Co-localization with MitoTracker (red) was observed. Merge images show the co-localization in yellow. DAPI was used to stain nucleus and kinetoplast (blue). DIC, differential interference contrast microscopy. (b) Antibodies against GFP detected unique bands of the expected sizes in parasite lysates. Antibodies against $\alpha 1$-tubulin were used to confirm equal loading. 22, epimastigotes carrying the pTREX-TCCyP22-GFP; T, epimastigotes carrying the pTREX-GFP empty vector, as a control; wt, wild-type epimastigotes. 
a
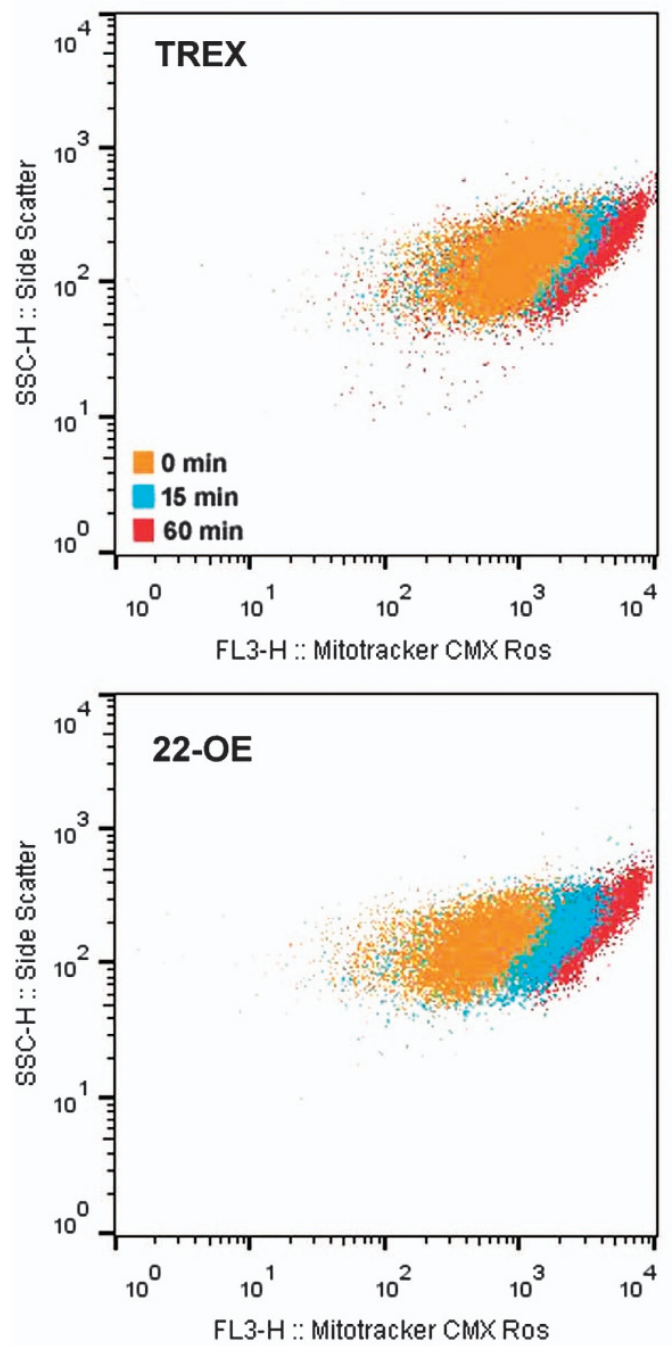

b

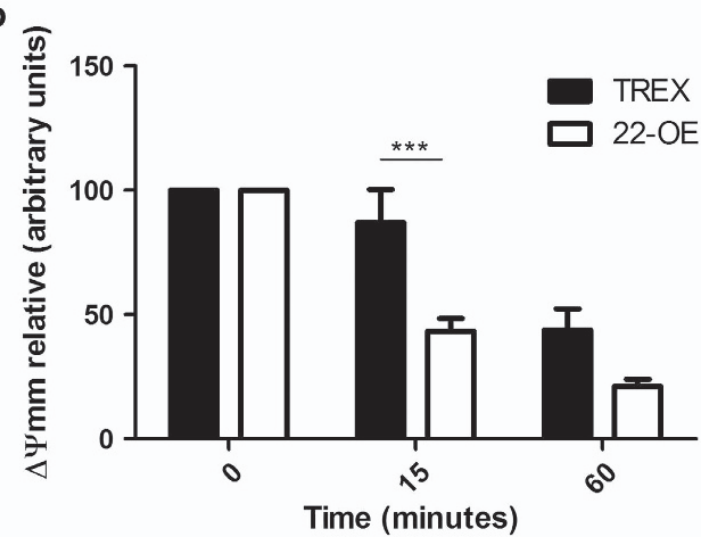

Figure 2. Loss of mitochondrial membrane potential $(\Delta \Psi \mathrm{mm})$ in oxidative stress conditions. Parasites were loaded with the $\Delta \Psi \mathrm{mm}$ fluorescent indicator, MitoTracker. Oxidative stress conditions were generated with $5 \mathrm{mM} \mathrm{H} \mathrm{H}_{2}$. At 15 and $60 \mathrm{~min}$, parasites were analyzed by flow cytometry. In (a), dot plots of a representative experiment are shown. (b) Quantification of three independent experiments. TREX, Control parasites carrying the empty pTREXeGFP vector; 22-OE, transgenic parasites overexpressing TCCyP22GFP (means \pm s.d., $n=3,{ }^{* * *} P<0.001$ (22-OE versus TREX at $15 \mathrm{~min}$ ), two-way - RM - ANOVA). a
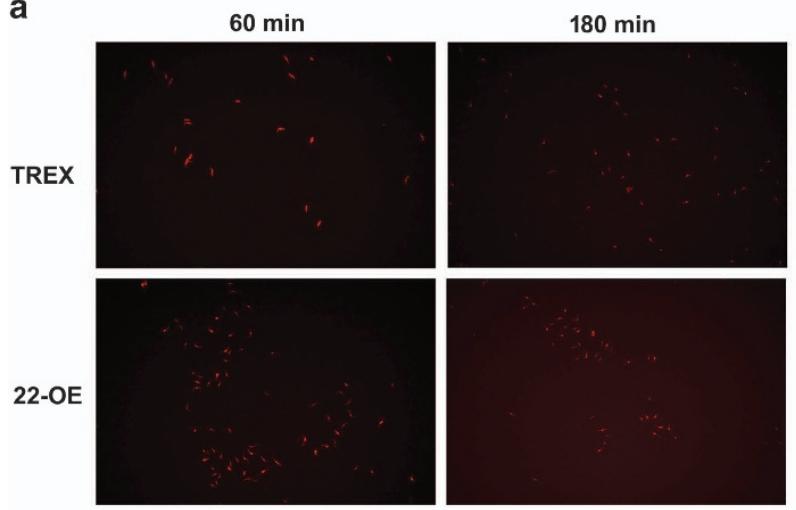

b

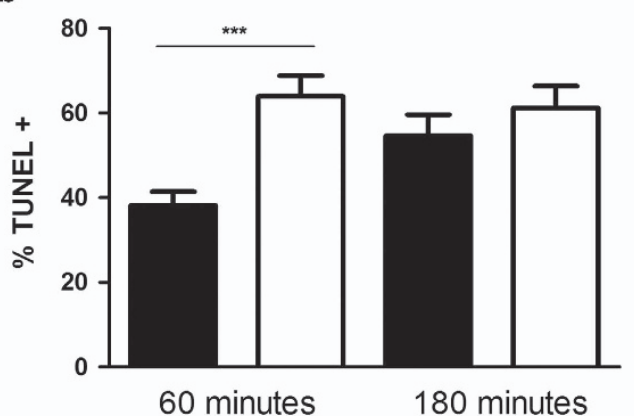

TREX

22-OE

Figure 3. DNA damage in oxidative stress conditions. Oxidative stress conditions were generated with $5 \mathrm{mM} \mathrm{H}_{2} \mathrm{O}_{2}$. At 60 and $180 \mathrm{~min}$, parasites were fixed and stained for TUNEL, according to manufacturers' specifications. (a) Fluorescence images of a representative experiment. TUNEL ${ }^{+}$parasites present nuclei with red staining. (b) Quantification of $\mathrm{TUNEL}^{+}$parasites, expressed as percentage over total parasites for each condition. TREX, Control parasites carrying the empty pTREX-eGFP vector; $22-\mathrm{OE}$, transgenic parasites overexpressing TcCyP22-GFP (means \pm s.d., $n=3$, ${ }^{* * *} P<0.001$ (22-OE versus TREX at $60 \mathrm{~min}$ ), Student's $t$-test).

percentage of TUNEL $^{+}$than TREX $(63,93 \%$ versus $38,18 \%$, respectively). However, the percentage of $\mathrm{TUNEL}^{+}-\mathrm{TCCyP} 22-\mathrm{OE}$ remained higher than TUNEL ${ }^{+}$-TREX control parasites, the difference was not significant after 180 min (Figure 3).

An increased number of TCCyP22-OE parasites presents loss of plasma membrane integrity

To evaluate another cell death feature, we used propidium iodide $(\mathrm{Pl})$, which positively stains the parasites that have lost their plasma membrane integrity. At different time points, parasites were washed to remove $\mathrm{H}_{2} \mathrm{O}_{2}$, stained with $\mathrm{PI}$ and analyzed by flow cytometry. The fluorescence intensity was used to identify $\mathrm{Pl}^{+}$parasites.

Since loss of membrane integrity is an event that seems to occur at the end of cell death process, after $60 \mathrm{~min}$ under oxidative stress stimulus only a low percentage of $\mathrm{Pl}^{+}$parasites were observed, although significantly higher in TcCyP22-OE than in TREX control parasites (12 and 6\%, respectively). After $180 \mathrm{~min}$, the proportion of $\mathrm{PI}^{+}-\mathrm{TCC}$ P $22-\mathrm{OE}$ parasites increased, reaching $44.55 \%$ of the population, compared with $14.35 \%$ in TREX control parasites (Figure 4), indicating that TcCyP22-OE parasites present a higher sensitivity to the oxidative stress environment.

Phospatidylserine exposure is not a significant cell death feature neither in TcCyP22-OE or TREX control parasites

With the aim of distinguishing the cell death process that occurs in response to $\mathrm{H}_{2} \mathrm{O}_{2}$ in $T C \mathrm{C} y \mathrm{P} 22-\mathrm{OE}$ parasites, we measured 
a
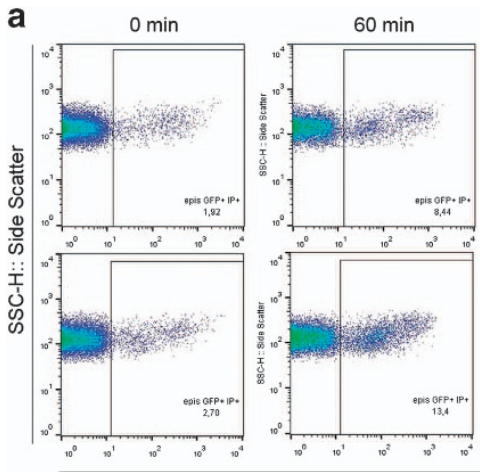

FL3-H:: Propidium lodide
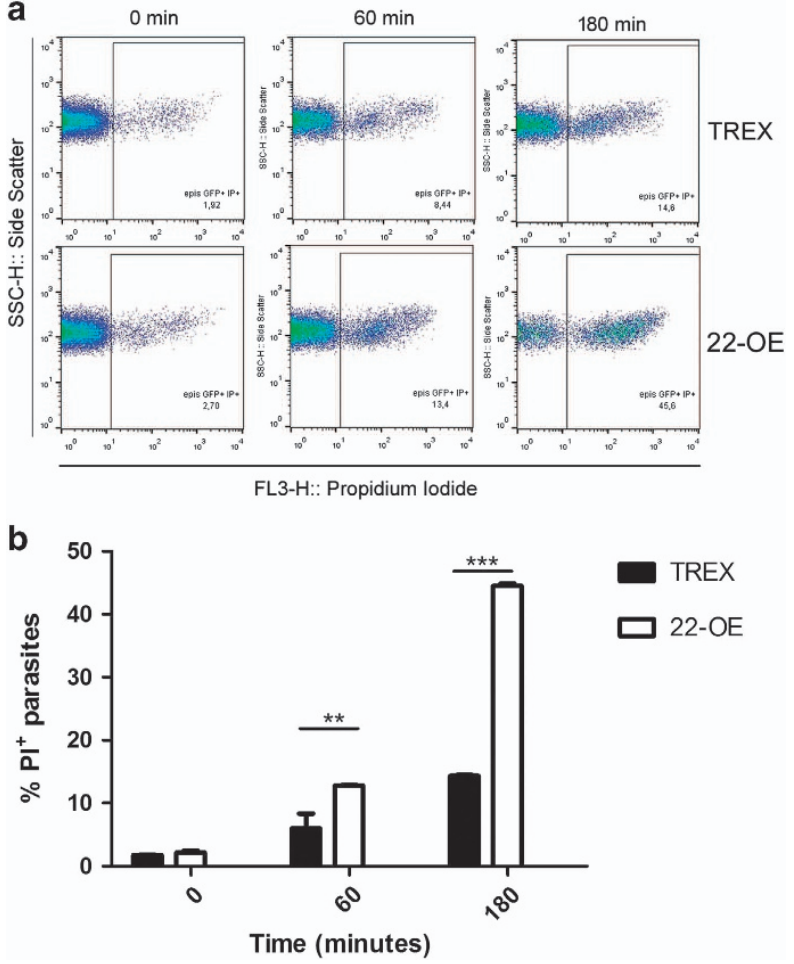

Figure 4. Loss of plasmatic membrane integrity during oxidative stress conditions. Parasites were incubated with $5 \mathrm{mM} \mathrm{H}_{2} \mathrm{O}_{2}$. At 60 and $180 \mathrm{~min}$, parasites were stained with propidium iodide and analyzed by flow cytometry. In (a), dot plots of a representative experiment are shown. (b) Quantification of three independent experiments. TREX, Control parasites carrying the empty pTREXeGFP vector; 22-OE, transgenic parasites overexpressing TcCyP22GFP (means \pm s.d., $n=3,{ }^{* * P}<0.01$ (22-OE versus TREX at $60 \mathrm{~min}$ ); ${ }^{* * * P}<0.001$ (22-OE versus TREX at $180 \mathrm{~min}$ ), Two-way - RM ANOVA).

phosphatidylserine (PS) exposure on the external leaflet of the plasma membrane. This event is evidenced by the annexin $\mathrm{V}$ binding assay to detect apoptotic cell death. Herein, we used a double staining procedure, which consists in using annexin $\mathrm{V}$ conjugated to phycoerythrin (AV-PE) to detect PS exposure and the fluorescent 7-aminoactinomycin D (7-AAD), for plasma membrane integrity, that allows the identification of different sub-populations: $A V-P E^{+} 7 A A D$, considered as early apoptotic parasites; AV-PE $7 A A^{+}$as necrotic ones. However, double positive $\mathrm{AV}^{-\mathrm{PE}^{+}} 7 \mathrm{AAD}^{+}$parasites cannot be unequivocally distinguished as late apoptotic or necrotic.

When oxidative stress was generated with $5 \mathrm{mM} \mathrm{H}_{2} \mathrm{O}_{2}$, no significant $\mathrm{AV}^{-\mathrm{PE}^{+}}$staining was observed for TCCyP22-OE or TREX parasites, indicating that an apoptotic-like cell death would not be occurring in these parasites, under the conditions assayed. However, we were able to detect $7 A A D^{+}$parasites (either simple or double positives), which indicates the plasma membrane integrity was lost. According to the results obtained with PI staining in parasites incubated with $\mathrm{H}_{2} \mathrm{O}_{2}$ for 180 min (Figure 4), TCCyP22-OE showed a significantly higher percentage of 7-AAD ${ }^{+}$ sub-population $(37.7+20.23 \%)$ than TREX control parasites (12.7+8.23\%; Figure 5).

The results described in this work suggest that TcCyP22-OE parasites undergo a regulated necrosis rather than an apoptoticlike cell death, in response to hydrogen peroxide. Under the conditions described, the parasites overexpressing the mitochondrial cyclophilin TcCyP22 presented an enhanced sensitivity to oxidative stress environment.

\section{DISCUSSION}

Cyclophilin D (CyPD) is a peptidyl prolyl isomerase (PPlase) localized to the mitochondrial matrix of mammalian cells. CyPD has been widely studied as the central component of the mitochondrial permeability transition pore (mPTP) in mammalian cells, a key channel that is formed during stress conditions such as $\mathrm{Ca}^{2+}$ overload and increased ROS production and that is capable of triggering a peculiar process of regulated necrosis which critically relies on CyPD. ${ }^{4}$ Although the specific molecular structure of the MPTP remains under discussion, CyPD has been the unique component proven to be indispensable, either in vivo, in animals with CyPD genetic ablation (Ppif ${ }^{-1}$ ) and in vitro, with CyPD pharmacological inhibition with cyclosporin $\mathrm{A}$.

As it was mentioned above, our research group has described the T. cruzi CyP gene family and reported the expression of several parasite cyclophilins that exhibited enzymatic PPlase activity, inhibited by $\mathrm{CsA}^{16,17}$ Recently, we observed that parasites submitted to an oxidative stress environment with $5 \mathrm{mM} \mathrm{H}_{2} \mathrm{O}_{2}$, underwent cell death events such as DNA degradation, ROS production, cytochrome $c$ translocation, which occurred after induction and were sensitive to CSA inhibition, suggesting that a T. cruzi mitochondrial cyclophilin could be present in a MPTP-like in this protozoan. ${ }^{19}$

In the present report, we identified that a homolog of mammalian cyclophilin D is expressed in T. cruzi, named as TcCyP22. This protein was localized to parasite mitochondrion in three stages of the parasite life cycle as expected, and to our knowledge, this is the first identification of a homolog of a CyPD in a protozoan parasite.

An overexpression of TcCyP22 cyclophilin in the epimastigote stage (TCCyP22-OE parasites) was our strategy to study the role of this protein in oxidative stress conditions, evaluating different known cell death features.

The loss of the mitochondrial membrane potential $\left(\Delta \Psi_{\mathrm{m}}\right)$ occurs at the onset of a cell death process and was measured in TcCyP22-OE parasites during oxidative stress; TREX parasites, carrying the empty pTREX-GFP vector, were used as control parasite population.

The observation that the loss of $\Delta \Psi_{\mathrm{m}}$ was significantly increased in TcCyP22-OE parasites with respect to TREX control parasites, when measured at 15 and $60 \mathrm{~min}$ after the addition of $5 \mathrm{mM} \mathrm{H} \mathrm{H}_{2}$ (Figure 2), indicates that the overexpression of TcCyP22 resulted in an enhanced sensitivity of parasites to the oxidative stress stimulus. In $T$. cruzi, the loss of $\Delta \Psi_{\mathrm{m}}$ in response to oxidative stress had already been studied using the fluorescent indicators $\mathrm{JC} 1^{\text {(ref. } 20)}$ and safranine-O. ${ }^{20,21}$ However, in our experimental model, these dyes could not be used, given the overlap with GFP signal.

Oxidative DNA damage is an unavoidable consequence of cellular metabolism. However, in cells undergoing oxidative stress caused by exogenous compounds, DNA damage can arise through overproduction of ROS and when lesions cannot be removed, chronic DNA damage triggers specific cell death responses such as apoptosis ${ }^{22}$ or some type of regulated necrosis. $^{4}$

When TcCyP22-OE parasites were exposed to $5 \mathrm{mM} \mathrm{H}_{2} \mathrm{O}_{2}$, a significantly higher DNA damage could be observed after $60 \mathrm{~min}$ by TUNEL technique, compared with the DNA damage observed in TREX control parasites (Figure 3 ).

DNA damage has also been reported to occur in other protozoan parasites, as Leishmania donovani under oxidative stress caused by $3 \mathrm{mM} \mathrm{H}_{2} \mathrm{O}_{2}{ }^{\text {(ref. 23,24) }}$ and in T. cruzi, with $20 \%$ (V/V) fresh human serum, ${ }^{20}$ and with of $5 \mathrm{mM} \mathrm{H}_{2} \mathrm{O}_{2}$ in our previous study. $^{19}$

Regarding parasite viability, during the whole oxidative stress induction, TcCyP22-OE presented an enhanced sensitivity to $\mathrm{H}_{2} \mathrm{O}_{2}$ stimulus in comparison with TREX control parasites. A significantly 
a

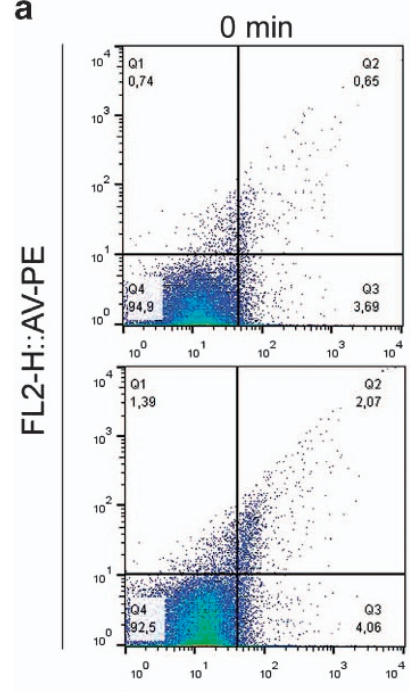

$60 \mathrm{~min}$

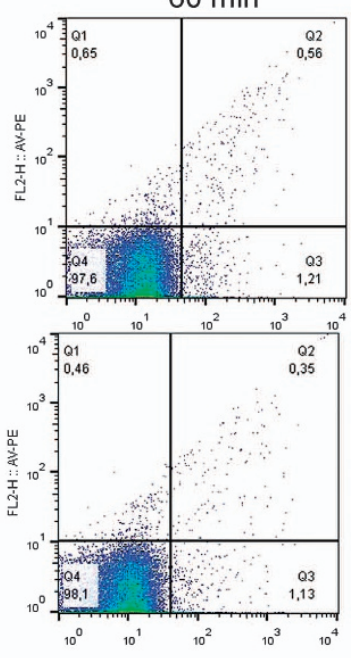

$180 \mathrm{~min}$

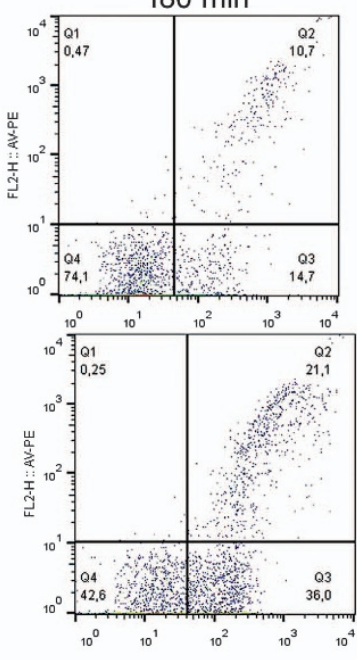

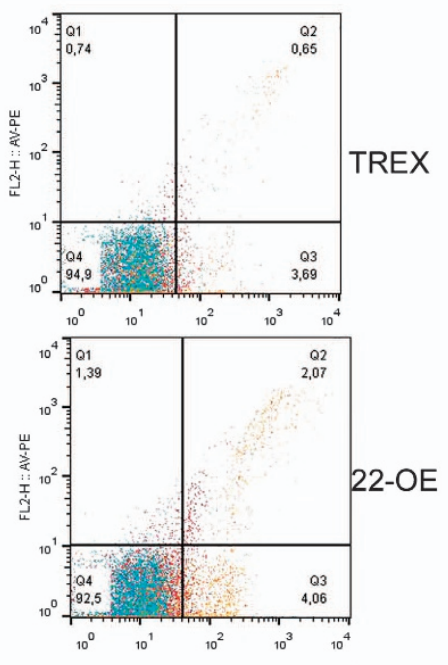

- Control

- $60 \mathrm{~min}$

$=180 \mathrm{~min}$
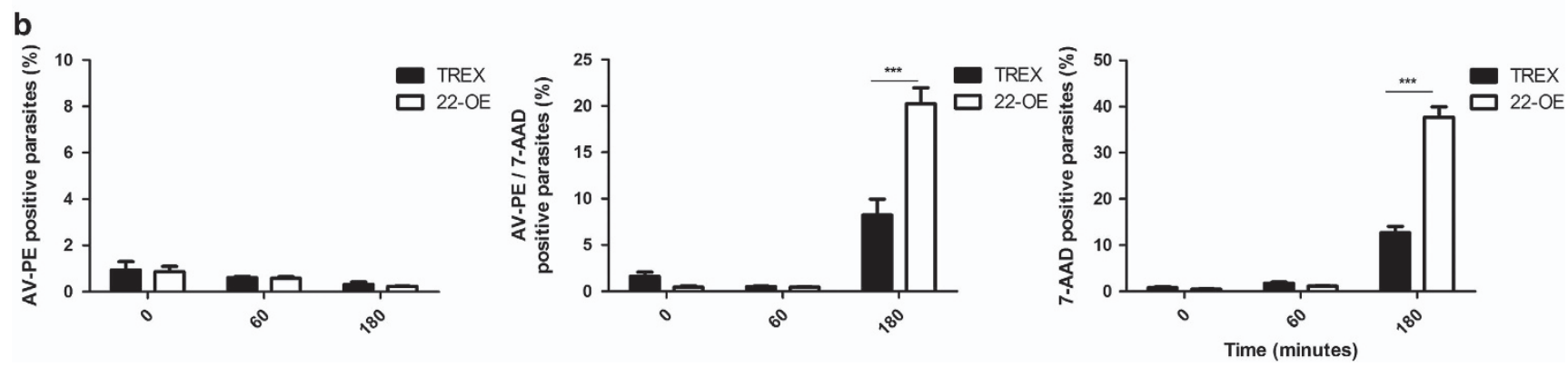

Figure 5. Phosphatidylserine exposure during oxidative stress. Parasites were incubated with $5 \mathrm{mM} \mathrm{H}_{2} \mathrm{O}_{2}$. At 60 and 180 minutes, parasites were stained with annexin V-phicoeritrin and 7AAD and analyzed by flow cytometry. Heat-shock treated parasites were used as positive control. In (a), dot plots of a representative experiment are shown. (b) Quantification of three independent experiments. TREX, Control parasites carrying the empty pTREX-eGFP vector; 22-OE, transgenic parasites overexpressing TcCyP22-GFP ***P $<0.001$ (22-OE versus TREX at $180 \mathrm{~min})$, two-way - RM - ANOVA).

higher number of TcCyP22-OE parasites lost their plasma membrane integrity, evidenced by the two different fluorescent indicators assayed, propidium iodide (Figure 4) and 7-AAD (Figure 5).For example, after 180 min of oxidative stress induction, TcCyP22-OE parasites $\mathrm{Pl}^{+}$were $\sim 45 \%$ (Figure 4 ) and $58 \%$ could be detected with 7-AAD (Figure 5). In contrast, for TREX control parasites these percentages were significantly lower, being $~ 15 \%$ for $\mathrm{PI}^{+}$(Figure 4) and $\sim 20 \%$ with 7-AAD ${ }^{+}$(Figure 5). $\mathrm{PI}$ is normally used as the viability probe of choice in flow cytometry and strongly intercalates DNA depending on the degree of unwinding. However, the viability stain 7-AADis a fluorescence DNA-binding agent that intercalates between cytosine and guanine bases and has a high DNA-binding constant and a slow post-fixation and permeabilization dissociation rate..$^{23,24}$

With the aim of discriminating the cell death process that might be occurring in TcCyP22-OE, we intended to detect phosphatidylserine (PS) exposure, which is a hallmark of apoptotic cell death, using the annexin $\mathrm{V}$ binding (AV-PE), together with 7-AAD staining. However, no significant single $A V-P^{+}$parasites could be detected during the whole time of the oxidative stress assay, nor for TCCyP22-OE or TREX control parasites (Figure 5), thus indicating that an apoptotic-like cell death would not be the type of cell death occurring in these conditions.

In the literature, PS exposure has been reported in T. cruzi, with different approaches. For instance, PS exposure was positively detected after oxidative stress induction using 20\% (v/v) FHS, in Tulahuen strain, grown in $\mathrm{BHI} .{ }^{20}$ PS exposure was also detected in axenic cultures of Tulahuen, grown in Diamonds' medium ${ }^{25}$ and in our previous work, in Brener, grown in BHI culture medium. ${ }^{19}$

Nevertheless, when grown in LIT medium, PS exposure was only detected in the $\mathrm{Y}$ strain trypomastigote stage but not in epimastigotes and amastigotes, ${ }^{26}$ in agreement with our results with the same $T$. cruzi strain and medium culture.

Nowadays, the study of cell death pathways has become a complex area, where every cell death process appears to be overlapped with the others, rather than independent or nonconnected mechanisms. In metazoans, the signaling pathways leading to regulated necrosis exhibit a crosstalk with the molecular cascades that lead to apoptosis, either due to shared signal transducers or for the existence of negative feedback circuitries, where one cell death process (most often apoptosis) actively inhibits the other (most frequently necrosis). Regulated necrosis is not only closely interconnected with apoptosis and autophagy, but also exhibits an elevated degree of overlap among different necrotic sub-routines, which would explain why the inhibition of a single signaling pathway is not enough to provide sufficient cytoprotective effects. ${ }^{4}$

Regarding T. cruzi, it could be expected that cell death pathways carried out by this single-cell organism were less complex than in metazoans. However, diversity still can be found in this protozoan 
parasite. ${ }^{19-21,25}$ The co-existence of several, still undescribed mechanisms underlying the MPT caused by oxidative stress conditions in T. cruzi could explain the different outcomes for the variety of stimuli assayed.

Taken together, results shown in this work indicate that TCCyP22 is not only localized to parasite mitochondrion, as it was expected for a homolog gene of mammalian CyPD, but also that parasites overexpressing this mitochondrial cyclophilin displayed an enhanced sensitivity to oxidative stress conditions compared with TREX control parasites, which leads to increased cell death, as has been reported for mammalian systems with CyPD overexpression. ${ }^{7}$

Our results encourage further experiments to continue elucidating the role of TcCyP22 in the metabolism of this protozoan parasite and to expand the research to other kinetoplastids.

The characterization of the pathway involving cell death in trypanosomes will lead to important insights into the biology of these parasites, the evolution of cell death pathways, and ultimately novel targets for anti-parasitic intervention.

\section{MATERIALS AND METHODS}

\section{Bioinformatic analysis}

To identify the putative mitochondrially localized cyclophilin within the $T$. cruzi family, CyP protein sequences were analyzed using two different servers, iPSORT ${ }^{27,28}$ and MitoProtll v1.101, ${ }^{29}$ which are available on the web. iPSORT is a subcellular localization site predictor for $\mathrm{N}$-terminal sorting signals (http://ipsort.hgc.jp/). MitoProtll calculates the N-terminal protein region that can support a Mitochondrial Targeting Sequence and the cleavage site (https://ihg.gsf.de/ihg/mitoprot.html).

The TcCyPs identified as with the highest probability to be localized to the parasite mitochondrion were aligned with human CyPD protein sequence (GenBankAcc No. P30405) using ClustalW Multiple Alignment functions of BioEdit program (http://www.mbio.ncsu.edu/bioedit/bioedit. html).

\section{Cloning of TcCyP22-GFP}

To construct C-terminally enhanced GFP-tagged TcCyP22 for localization in $T$. cruzi, the full-length cDNA of TcCyP22 was amplified from $T$. cruzi genomic DNA ( $Y$ strain) by PCR using the forward primer $5^{\prime}-C C C$ TCTAGAATGTITTCTCGTACATGGTTITGGG-3' and the reverse primer $5^{\prime}$-CGAA GCTTGTTGTITITGACTTCACCACAGTCC- $3^{\prime}$ (where the underlined nucleotides indicate the introduced $X b a l$ and Hindlll restriction sites, respectively). The PCR product was digested with $\mathrm{Xbal}$ and HindIII and then cloned in frame into the insertion sites in the pTREX-eGFP vector. ${ }^{30}$ The double-stranded sequences of GFP-tagged TCCyP22 constructs were confirmed as correct by DNA sequencing. The pTREX-TcCyP22-GFP DNA was transfected into T. cruzi epimastigotes, Y strain by electroporation in Cytomix buffer at 1 . $5 \mathrm{kV}$ with 3 pulses $25 \mu \mathrm{F}$, $\infty$ resistance each and selected with $250 \mu \mathrm{g} / \mathrm{ml}$ G418 antibiotic, for 2 weeks approximately. Enrichment of parasites expressing TcCYP22-GFP or GFP (from parasites carrying the empty pTREXGFP, used as control parasites) was performed by $\mathrm{GFP}^{+}$sorting using a Bio-Rad S3 cell sorter, Hercules, CA, USA.

\section{Parasite culture}

Trypanosoma cruzi epimastigotes, $\mathrm{Y}$ strain, were grown at $28^{\circ} \mathrm{C}$ in LIT medium ${ }^{31}$ supplemented with $10 \%$ fetal bovine serum (FBS) and $250 \mu \mathrm{g} / \mathrm{ml}$ G418 for pTREX selection. For oxidative stress experiments, epimastigotes were collected in log phase, at 3 days of growth. For T. cruzi trypomastigote and amastigote production, a monolayer of VERO cells was co-cultivated with a late log phase epimastigote suspension ( $>6$ days), where some parasites in trypomastigote stage were spontaneously detected, using RPMl supplemented with 10\% FBS. After 15 days, $T$. cruzi trypomastigotes and amastigotes were obtained from the supernatant. TcCyP22-GFP expression was confirmed by immunofluorescence.

\section{Immunofluorescence microscopy}

To determine the localization and expression of TCCyP22 in T. cruzi, live parasites (epimastigote, trypomastigote or amastigote stage) were labeled for 30 min with Mitotracker Red CMXRos (Invitrogen, Waltham, MA, USA) at
$50 \mathrm{nM}$ in LIT culture medium. Parasites were washed in PBS and fixed with $4 \%$ paraformaldehyde in PBS at room temperature for $1 \mathrm{~h}$. The fixed parasites were washed twice with PBS, allowed to adhere to poly-L-lysinecoated coverslips and permeabilized with $0.3 \%$ Triton X-100/PBS for 3 min. After blocking with PBS containing 3\% BSA, 1\% fish gelatin, $50 \mathrm{mM}$ $\mathrm{NH}_{4} \mathrm{Cl}$ and $5 \%$ goat serum for $1 \mathrm{~h}$, parasites were stained in 3\% BSA/PBS with the rabbit antibody against GFP (1:1000, Thermo Fisher Scientific, Waltham, MA, USA), for $1 \mathrm{~h}$. After thoroughly washing with PBS containing 3\% BSA, parasites were incubated with Alexa 488-conjugated goat anti-rabbit antibody at 1:1000 for $1 \mathrm{~h}$. The parasites were counterstained with 40,6-diamidino-2-phenylindole before mounting with GoldProLong Gold antifade reagent (Molecular Probes, Waltham, MA, USA). Differential interference contrast and fluorescent optical images were captured using an Olympus IX-71inverted fluorescence microscope with a Photometrix Cool Snap HQ charge-coupled device camera driven by DeltaVision software (Applied Precision, Fairfield, CT, USA).

\section{Western blot detection of TcCyp22-GFP}

T. cruzi lysatesfrom TCCyP22-OE, TREX or wild type parasites were separated by SDS-PAGE, carried out as described. ${ }^{32}$ Proteins were electrotransferred from $13.5 \%$ polyacrylamide gels to nitrocellulose membranes, which were blocked with a 5\% (W/V) non-fat milk suspension for $1 \mathrm{~h}$ at room temperature. After incubation for $1 \mathrm{~h}$ with a rabbit monoclonal antibody against GFP (1:1000, Roche, Mannheim, Germany) and a 1:3000 dilution of the goat anti-rabbit immunoglobulin $G$ conjugated with horseradish peroxidase (Jackson Laboratories, West Grove, PA, USA), proteins were visualized by chemiluminescence with an ECL Western Blotting Detection kit. For equal loading control, membranes were stripped and re-blotted with a rabbit polyclonal antibody against a1-tubulin (1:3000; Santa Cruz Biotechnology, Dallas, TX, USA).

\section{Induction of oxidative stress}

Epimastigotes in log phase of growth were collected and washed twice with PBS-3\% glucose to remove culture medium. Parasites were resuspended to $1 \times 10^{6}$ epimastigotes/ml in PBS-3\% glucose and oxidative

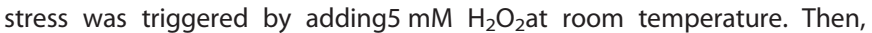
parasites were centrifuged at $2300 \mathrm{rpm}$ for $10 \mathrm{~min}$ and washed once in PBS-3\% glucose. Pellets were resuspended in PBS-3\% glucose for subsequent studies.

\section{Mitochondrial membrane potential $(\Delta \Psi \mathrm{m})$}

Epimastigotes in a cell density of $\sim 1 \times 10^{6}$ parasites $/ \mathrm{ml}$ were loaded with the fluorescent $\Delta \psi_{\mathrm{m}}$ indicator Mitotracker Red CMXRos (100 nM, Molecular Probes, Invitrogen) in LIT medium, for $30 \mathrm{~min}$ at $28^{\circ} \mathrm{C}$ with gentle agitation. Then, parasites were washed twice with PBS-3\% glucose and resuspended again in $1 \times 10^{6}$ parasites per $\mathrm{ml}$ to proceed to oxidative stress incubation. Fluorescence was detected in a FACS Calibur equipment (Becton Dickinson and Co., Franklin Lakes, NJ, USA), were GFP fluorescence was detected in FL1-1 photodetector; and MitoTracker in FL-3. Data were analyzed using FlowJo10 software (Ashland, OR, USA). Results are expressed as the mean of fluorescence intensity of parasite population for each condition (50.000 $\mathrm{GFP}^{+}$events were considered as 100\%).

\section{In situ labeling of DNA fragments (TUNEL)}

To evaluate DNA integrity after oxidative stress treatment, $1 \times 10^{6}$ epimastigoteswere collected, washed twice and resuspended inPBS-3\% glucose. After oxidative stress induction (as in 2.6), in situ detection of DNA fragments by TUNEL was performed using the In Situ Cell Death detection kit (Roche). Epimastigotes were collected, fixed in $4 \%$ formaldehyde and coated onto poly-L-lysine covered slides. Permeabilization was done with $0,1 \%(\mathrm{v} / \mathrm{v})$ Triton X-100/PBS for $5 \mathrm{~min}$ at room temperature, followed by incubation with TdT buffer containing nucleotide $\mathrm{mix}(50 \mathrm{mM}$ tetramethylrhodamine- 12-dUTP, $100 \mathrm{mMd}$ ATP, $10 \mathrm{mM}$ Tris-HCl, $1 \mathrm{mM}$ EDTA, $\mathrm{pH}$ 7.6) for $1 \mathrm{~h}$ at $37^{\circ} \mathrm{C}$. The samples were visualized under a fluorescence microscope. The percentage of parasites showing a clearly visible nuclear staining was determined in TCCyP22-OE and TREX control parasites in different conditions by counting 100 cells in triplicate (blind-coded samples). 
FACS Analysis for Cell viability and Detection of Phosphatidylserine (PS) exposure

After oxidative stress incubation, $1 \times 10^{6}$ epimastigotes were collected at different time points $(0,60$ or $180 \mathrm{~min})$ as in Induction of oxidative stress.

Cell viability

parasites were stained with $0,5 \mu \mathrm{g} / \mathrm{ml}$ propidium iodide (PI). GFP fluorescence was detected in FL1-1 photodetector; PI, in FL-3. Results were expressed as the percentage of $\mathrm{PI}^{+}$positive parasites with respect to the total parasite count in each condition.

\section{PS exposure}

PS on parasites external surface of the plasma membrane was detected using the Annexin V: PE Apoptosis Detection Kit (Becton Dickinson and Co.) according to the manufacturer's protocol. Co-staining of the parasites with 7-aminoactinomycin $D(7-A A D)$ was performed, to evaluate the integrity of plasma membrane. Heat-shock treated parasites $\left(3 \mathrm{~min}\right.$ at $80^{\circ} \mathrm{C}$ ) were used as positive control for AV-PE and 7-AAD staining. GFP fluorescence was detected in FL1-1 photodetector; PE, in FL-2 and 7-AAD in FL-3.Results were expressed as the percentage of positive parasites in each quadrant with respect to the total parasite count in each condition.

Fluorescence was detected in a FACS Calibur equipment (Becton Dickinson and Co. TreeStar Inc, Ashland, OR, USA). Data analyzed using FlowJo10 software with $50000 \mathrm{GFP}^{+}$events were considered as $100 \%$.

\section{ACKNOWLEDGEMENTS}

We thank Melisa Sayé, Chantal Reigada, Dr Mariana Miranda and Dr Claudio Pereira for their help with fluorescence microscopy. We are grateful for the valuable help of Dr Silvia Moreno and Dr Roberto Docampo laboratories during PLB's research visit to the CTEGD, University of Georgia, financed by a scholarship from Fulbright Commission and Fundación Bunge y Born. This work was supported by ANLIS Carlos G Malbrán, PICTO-ANLIS 2011 and Focanlis 2014.

\section{COMPETING INTEREST}

The authors declare no conflict of interest.

\section{REFERENCES}

1 Javadov S, Kuznetsov A. Mitochondrial permeability transition and cell death: the role of cyclophilin d. Front Physiol 2013; 4: 762013.

2 Kroemer G, Galluzzi L, Brenner C. Mitochondrial membrane permeabilization in cell death. Physiol Rev 2007; 87: 99-163.

3 Brenner C, Grimm S. The permeability transition pore complex in cancer cell death. Oncogene 2006; 25: 4744-4756.

4 Galluzzi L, Kepp O, Krautwal S, Kroemer G, Linkermann A. Molecular mechanisms of regulated necrosis. Semin Cell Dev Biol 2014; 35: 24-32.

5 Tait SW, Green DR. Mitochondria and cell death: outer membrane permeabilization and beyond. Nat Rev Mol Cell Biol 2010; 11: 621-632.

6 Galluzzi L, Kepp O, Kroemer G. Mitochondria: master regulators of danger signalling. Nat Rev Mol Cell Biol 2012; 13: 780-788.

7 Baines CP, Kaiser RA, Purcell NH, Blair NS, Osinska H, Hambleton MA et al. Loss of cyclophilin $D$ reveals a critical role for mitochondrial permeability transition in cell death. Nature 2005; 434: 658-662.

8 Nakagawa T, Shimizu S, Watanabe T, Yamaguchi O, Otsu K, Yamagata H et al. Cyclophilin D-dependent mitochondrial permeability transition regulates some necrotic but not apoptotic cell death. Nature 2005; 434: 652-658.

9 Kung G, Konstantinidis K, Kitsis RN. Programmed necrosis, not apoptosis, in the heart. Circ Res 2011; 108: 1017-1036.

10 Schinzel AC, Takeuchi O, Huang Z, Fisher JK, Zhou Z, Rubens J et al. CyclophilinD is a component of mitochondrial permeability transition and mediates neuronal cell death after focal cerebral ischemia. Proc Natl Acad Sci USA 2005; 102 12005-12010.

11 Vaseva AV, Marchenko ND, Ji K, Tsirka SE, Holzmann S, Moll UM. p53 opens the mitochondrial permeability transition pore to trigger necrosis. Cell 2012; 149: 1536-1548.
12 Linkermann A, Brasen JH, Darding M, Jin MK, Sanz AB, Heller JO et al. Two independent pathways of regulated necrosis mediate ischemia-reperfusion injury. Proc Natl Acad Sci USA 2013; 110: 12024-12029.

13 Devalaraja-Narashimha K, Diener AM, Padanilam BJ. Cyclophilin D gene ablation protects mice from ischemic renal injury. Am J Physiol Renal Physiol 2009; 297: F749-F759.

14 Blumental S, Lambermont M, Heijmans C, Rodenbach MP, El Kenz H, Sondag D et al. First documented transmission of Trypanosoma cruzi infection through blood transfusion in a child with sickle-cell disease in Belgium. PLoS Negl Trop Dis 2015; 9: e0003986.

15 WHO 2016. http://www.who.int/mediacentre/factsheets/fs340/en/.

16 Potenza M, Galat A, Minning TA, Ruiz AM, Duran R, Tarleton RL et al. Analysis of the Trypanosoma cruzi cyclophilin gene family and identification of cyclosporine $A$ binding proteins. Parasitology 2006; 132: 867-882.

17 Bua J, Fichera LE, Fuchs AG, Potenza $M$, Dubin $M$, Wenger RO et al. Anti-Trypanosoma cruzi effects of cyclosporin A derivatives: possible role of a P-glycoprotein and parasite cyclophilins. Parasitology 2008; 135: 217-228.

18 Galat A, Bua J. Molecular aspects of cyclophilins mediating therapeutic actions of their ligands. Cell Mol Life Sci 2010; 67: 3467-3488.

19 Bustos PL, Perrone AE, Milduberger N, Postan M, Bua J. Oxidative stress damage in the protozoan parasite Trypanosoma cruzi is inhibited by cyclosporin A Parasitology 2015; 142: 1024-1032.

20 Piacenza L, Irigoín F, Alvarez MN, Peluffo G, Taylor MC, Kelly JM. Mitochondrial superoxide radicals mediate programmed cell death in Trypanosoma cruzi: cytoprotective action of mitochondrial iron superoxide dismutase overexpression. Biochem J 2007; 403: 323-334.

21 Fernandes MP, Leite ACR, Araujo FFB, Saad STO, Baratti MO, Correia MTS et al. The Cratyliamollis seed lectin induces membrane permeability transition in isolated rat liver mitochondria and a cyclosporine A-insensitive permeability transition in Trypanosoma cruzi mitochondria. J Eukaryotic Microbiol 2014; 61: 381-388.

22 Norbury C, Zhivotovsky B. DNA damage-induced apoptosis. Oncogene 2004; 23 2797-2808.

23 Das M, Mukherjee SB, Shaha C. Hydrogen peroxide induces apoptosis-like death in Leishmaniadonovanipromastigotes. J Cell Sci 2001; 114: 2461-2469.

24 Falzone N, Huyser C, Franken DR. Comparison between propidium iodide and 7-amino-actinomycin-D for viability assessment during flow cytometric analyses of the human sperm acrosome. Andrologia 2010; 42: 20-26.

25 Jimenez V, Paredes R, Sosa MA, Galanti N. Natural programmed cell death in T. cruzi epimastigotes maintained in axenic cultures. J Cell Biochem 2008; 105 688-698.

26 DaMatta R, Seabra S, Deolindo P, Arnholdt A, Manha L, Goldenberg S et al. Trypanosoma cruzi exposes phosphatidylserine as an evasion mechanism. FEMS Microbiol Lett 2007; 266: 29-33.

27 Bannai H, Tamada Y, Maruyama O, Nakai K, Miyano S. Extensive feature detection of N-terminal protein sorting signals. Bioinformatics 2002; 18: 298-305.

28 Bannai H, Tamada Y, Maruyama O, Nakai K, Miyano S. Views: fundamental building blocks in the process of knowledge discovery. In: Proceedings of the 14th International FLAIRS Conference. AAAI Press: Florida, USA, 2001, pp 233-238.

29 Claros MG, Vincens P. Computational method to predict mitochondrially imported proteins and their targeting sequences. Eur J Biochem 1996; 241: 770-786.

30 Vazquez Martin P, Levin Mariano J. Functional analysis of the intergenic regions of TcP2b gene loci allowed the construction of an improved Trypanosoma cruzi expression vector k. Gene 1999; 239: 217-225.

31 Camargo EP. Growth and differentiation in Trypanosoma cruzi I. Origin of metacyclic trypanosomes in liquid media. Rev Inst Med Trop Sao Paulo 1964; 6: 93-100.

32 Laemmli UK. Cleavage of structural proteins during the assembly of the head of bacteriophage T4. Nature 1970; 227: 680-685.

This work is licensed under a Creative Commons Attribution 4.0 International License. The images or other third party material in this article are included in the article's Creative Commons license, unless indicated otherwise in the credit line; if the material is not included under the Creative Commons license, users will need to obtain permission from the license holder to reproduce the material. To view a copy of this license, visit http://creativecommons.org/licenses/ by/4.0/

(c) The Author(s) 2017

Supplemental Information accompanies the paper on the Cell Death and Discovery website (http://www.nature.com/cddiscovery) 